\title{
Effect of the Antibody Immunotherapy by the Anti-MUC1 Monoclonal Antibody to the Oral Squamous Cell Carcinoma in Vitro
}

\author{
Momoko SHIMIzu $^{a}$ and Masaki IMAI ${ }^{*}, b$ \\ ${ }^{a}$ First Department of Oral and Maxillofacial Surgery, Osaka Dental University; 8-1 Kuzuhahanazono-cho, Hirakata, \\ Osaka 573-1121, Japan: and ${ }^{b}$ Department of Immunology, Graduate School of Medical Sciences, Nagoya City University; \\ 1 Kawasumi, Mizuho-cho, Mizuho-ku, Nagoya 467-8601, Japan. \\ Received May 9, 2008; accepted October 2, 2008; published online October 6, 2008
}

To conserve both form and function in the oral area, effective and selective drugs against oral cancer will be required. We focused on MUC1, a transmembrane glycoprotein, that is considered tumor-associated antigens (TAAs) for cancer therapy. Recently, studies were done to evaluate the patterns of MUC1 expression in oral squamous cell carcinomas (OSCCs) and it was found that higher MUC1 expression correlates with tumor invasion and metastasis. Using oral squamous cell carcinoma cell lines, we demonstrate here that tumor-specific targeting of MUC1 with the specific monoclonal antibody $\mathrm{C} 595$ has functional consequences with regard to complement deposition on MUC1-expressing oral cancer cell lines. Anti-MUC1 monoclonal antibody (mAb) also induced complement-dependent cytotoxicity (CDC) and antibody-dependent cellular cytotoxicity (ADCC) to OSCC cells, and these effects were strongly correlated with MUC1 expression. Thus, these results indicate that anti-MUC1 mAb could provide a useful tool against OSCCs, and may provide insight into the development of low side-effect targeting therapy for this malignant disease.

Key words oral squamous cell carcinoma; antibody; immunotherapy; human mucin 1

In 2007 in United States, oral squamous cell carcinomas (OSCCs) were estimated to be the eighth leading cause of cancer, accounting for $3 \%$ of malignancies in men. ${ }^{1)}$ At present, surgery and radiation therapy, separately or in combination, are the main courses of treatment. In advanced stages of the disease, chemotherapy is added to surgery and/or radiation. ${ }^{1)}$ However, preservation of organ structure and function and fewer therapeutic side effects in the oral area are important for the treatment of oral cancer, because some patients worry that they may become functionally and cosmetically unacceptable. In addition, OSCC is resistant to both radiation $^{2,3)}$ and chemotherapy. ${ }^{3,4)}$ Thus, there are a number of problems associated with OSCC treatment. Recently, various basic and clinical observations suggest that the patient's immune system plays a role in the course of oral cell cancer. In vivo tumor regression by IL-12 and IL-2, inducing CD80 expression, ${ }^{5)}$ the presence of a $\mathrm{CD} 8^{+} \mathrm{T}$-cell-mediated immune response $^{6)}$ and the number of dendritic cells infiltrating the tumor are highly significant prognostic parameters. ${ }^{7)}$ These results have lead to the hypothesis that activation of the patient's immune system will enhance anti-OSCC therapy.

MUC1 (human mucin 1, epsialin) is an epithelial, cell-associated mucin normally expressed on the apical surface, but differences in MUC1 expression on cancer cells make MUC1 epitopes tumor-specific. On tumor cells, MUC1 is highly overexpressed and loses its polarized distribution on the cell surface. Tumor-associated MUC1 is also underglycosylated, resulting in the exposure of immunodominant peptide sequences that are normally concealed. MUC1 is expressed by most adenocarcinomas of the breast, lung, stomach, pancreas, colon, ovary, bladder and prostate. MUC1 is thus an important marker of malignancy and is a target for several immunotherapies currently under investigation (for reviews on MUC1 and cancer, see ref. 8). The level of MUC1 expression has been shown to correlate with the degree of breast tumor differentiation, estrogen receptor status, and clinical outcome of breast cancers, ${ }^{9}$ plus both progression and metastases of epithelial ovarian cancers. ${ }^{10)}$ AntiMUC1 C595 monoclonal antibody (mAb) recognizes a tumor-associated core protein epitope and currently investigates under the clinical studies. In OSCCs, distinct membrane MUC1 mucin staining patterns were identified in $59.7 \%$ of OSCC patients. ${ }^{11)}$ Furthermore, the malignant transformation of oral epithelium, tumor invasion, and tumor metastasis were associated with higher MUC1 mucin expression. ${ }^{11)}$ Thus, MUC1 mucin expression may be a useful diagnostic marker for prediction of the invasive/metastatic potential of OSCC.

Immunotherapy using mAbs holds great promise as an anti-cancer therapeutic strategy because of its ability to target cancer cells specifically while sparing the surrounding normal tissue. This is an important advantage over relatively non-specific chemo- and radio-therapeutic treatments. Eight anti-cancer mAbs have been approved by the Food and Drug Administration (FDA) and many more are in clinical development. ${ }^{12)}$ However, the antibody targeting for oral cancer (also head and neck cancer) remains to be discovered, although antibody immunotherapy for cancer has been reported frequently. In our study, the possibility of using the patient's own immune system to eliminate OSCCs was investigated using an anti-MUC1 $\mathrm{mAb}$. We also explored an antiMUC1 antibody to investigate therapeutic potential and functional mechanism(s) in a well-characterized in vitro model with common OSCC cell lines.

\section{MATERIALS AND METHODS}

Cell Lines and Cultures Human oral squamous cell carcinoma cell lines HO-1-u-1 and SAS cells were obtained from the Cell Resource Center for Biomedical Research, Tohoku University, and HSC3 and HSC4 cell lines were obtained from Riken Cell Bank. HSC3 cells were maintained in 
MEM (Wako, Osaka, Japan), and HO-1-u-1 and HSC4 cells were grown in RPMI1640 (Wako, Osaka, Japan). These media were supplemented with $10 \%$ fetal bovine serum (FBS; Hyclone Laboratories, Inc., Logan, UT, U.S.A.) and antibiotics (100 units $/ \mathrm{ml}$ penicillin and $100 \mu \mathrm{g} / \mathrm{ml}$ streptomycin; Invitrogen, La Jolla, CA, U.S.A.). The cells were cultured in a $5 \% \mathrm{CO}_{2}$ humidified incubator at $37^{\circ} \mathrm{C}$. SAS cells were grown in RPMI1640 with $10 \%$ heat-inactivated FCS, $100 \mathrm{units} / \mathrm{ml}$ penicillin, $100 \mathrm{mg} / \mathrm{ml}$ streptomycin, and $1 \mu \mathrm{g} / \mathrm{ml}$ fungizone (Invitrogen, La Jolla, CA, U.S.A.).

Monoclonal Antibodies and Polyclonal Serum Antibodies Anti-MUC1 mAb C595 (mouse IgG3) was purchased from Abcam (Tokyo, Japan). Anti-complement regulatory protein (CD46, CD55, and CD59) antibodies were obtained from BD Pharmingen (San Diego, CA, U.S.A.). FITC-goat anti-human C3 IgG and FITC-goat anti-mouse IgG were obtained from ICN Pharmaceuticals (Aurora, OH, U.S.A.) and all other secondary antibodies were purchased from Sigma (St. Louis, MO, U.S.A.). Mouse IgG1 and IgG3 isotype control antibodies were purchased from Santa Cruz Biotechnology Inc (Santa Cruz, CA, U.S.A.) and Ancell (Bayport, MN, U.S.A.), respectively. Normal human serum (NHS) was obtained from the blood of healthy volunteers in the laboratory. All serum was stored in aliquots at $-80^{\circ} \mathrm{C}$ until use.

Flow Cytometry Analysis of cell surface expression of MUC1 or complement regulatory proteins was performed as described. ${ }^{13)}$ For analysis of human C3 deposition on OSCC cells in vitro, cells $\left(5 \times 10^{5}\right)$ were resuspended in $20 \mu \mathrm{l} \mathrm{PBS}$ (Invitrogen, La Jolla, CA, U.S.A.) or anti-MUC1 mAb C595 at $100 \mu \mathrm{g} / \mathrm{ml}$ and incubated for $30 \mathrm{~min}$ at $4{ }^{\circ} \mathrm{C}$. Cells were then washed and incubated in $20 \mu \mathrm{l}$ of normal human serum diluted to the indicated concentration with Dulbecco's phosphate buffered saline (D-PBS) (Sigma, St. Louis, MO, U.S.A.) at $37^{\circ} \mathrm{C}$ for $30 \mathrm{~min}$. Cells were then washed with PBS four times, incubated with FITC-conjugated goat antihuman $\mathrm{C} 3\left(30 \mathrm{~min} / 4^{\circ} \mathrm{C}\right)$ and washed twice. Finally, cells were suspended in PBS containing $2 \mu \mathrm{g} / \mathrm{ml}$ propidium iodide (PI) and analyzed by flow cytometry. PI-positive (dead) cells were excluded when fluorescence intensity was calculated.

Detection of Complement Mediated Cytotoxicity For complement-dependent cytolysis assays, OSCC cells $\left(5 \times 10^{4}\right)$ were seeded with $50 \mu \mathrm{l}$ of the culture medium in flatbottom 96-well plates. Ten microliters of C595 mAb (final $100 \mu \mathrm{g} / \mathrm{ml}$ ) or PBS was then dispensed into the wells, and normal human serum was added to the individual wells at various concentrations from 0 to $40 \%$ to give a final volume of $100 \mu \mathrm{l}$. After incubation for $2 \mathrm{~h}$ at $37^{\circ} \mathrm{C}$, cell viability was determined by cell counting kit 8 (Dojindo laboratory, Kumamoto, Japan) according to the manufacturer's instructions. Lytic activity was calculated using the means of replicate wells according to the following formula: \% cell lysis $=100-[(a-c) /(b-c)] \times 100$, whereby $(a)$ is absorbance values of each sample, $(b)$ is absorbance values for the culture medium background, and $(c)$ is absorbance values after lysis with $1 \%$ Triton X-100.

Detection of Antibody Dependent Cellular Cytotoxicity (ADCC) ADCC was examined using the CytoTox 96 NonRadioactive Cytotoxicity Assay (Promega, Madison, WI, U.S.A.). For quantification of ADCC activity, peripheral blood mononuclear cells (PBMCs) were isolated from healthy volunteers with Ficoll-Paque Plus (GE Healthcare
UK Ltd., Little Chalfont, U.K.) and used as effector cells. The target cells were suspended in RPMI medium with $2 \%$ FBS and plated in a 96-well V-bottom microtiter plate at $1 \times 10^{4}$ cells/well. Effector cells were added at various effector-to-target cell ratios from $10: 1$ to $100: 1$ in the presence or absence of $50 \mu \mathrm{g} / \mathrm{ml}$ of C $595 \mathrm{mAb}$. The plates were incubated for $4 \mathrm{~h}$ at $37^{\circ} \mathrm{C}$, and the absorbance of the supernatants at $490 \mathrm{~nm}$ was recorded to determine the LDH release. The average of absorbance values for the culture medium background was subtracted from experimental release $(A)$, target cell spontaneous release $(B)$, effector cell spontaneous release $(C)$ and target cell maximum release $(D)$. The specific cytolysis percentage was calculated using the following formula:

\section{$\%$ cell lysis $=(A-B-C) /(D-B) \times 100$}

Cell Proliferation Assay Cell proliferation was determined by the MTS tetrazolium assay (CellTiter $96^{\circledR} \mathrm{AQ}_{\text {ueous }}$ One Solution Assay Kit, Promega, Madison, WI, U.S.A.) and enumeration after trypan blue staining. Both methods gave similar results. Cells $\left(4 \times 10^{5}\right.$ cells/well $)$ were plated in $96-$ well tissue culture plates in serum-containing medium and then treated with the indicated concentration of C595 mAb for $15 \mathrm{~h}$ at $37^{\circ} \mathrm{C}$. After incubation, $20 \mu \mathrm{l}$ of MTS was added into each well of the culture plate containing the samples (in $100 \mu \mathrm{l}$ of culture medium), and this plate was incubated for $1 \mathrm{~h}$ at $37^{\circ} \mathrm{C}$ in a humidified, $5 \% \mathrm{CO}_{2}$ atmosphere. The absorbances were determined with a plate reader at $490 \mathrm{~nm}$. All data represent the average values for a minimum of three wells and at least three trials. The percentage of living cells was calculated using the following formula: \% living cells $=(a-c) /(b-c) \times 100$, whereby $(a)$ is absorbance values of each sample, $(b)$ is absorbance values for the PBS-treated negative controls, and $(c)$ is absorbance values after lysis with $1 \%$ Triton X-100 as $100 \%$ cell death-positive controls.

Statistical Analysis Unpaired Welch's $t$ tests were used to determine statistical differences. The significance of the correlations was determined by means of the Pearson $R$ test. Significance was accepted at the $p<0.05$ level.

\section{RESULTS}

Tumor-Associate Antigen MUC1 Expression on Human Oral Squamous Cell Carcinomas Cell Lines Immunohistochemical studies were previously reported showing that $59.7 \%$ of the OSCC patients were positive for MUC1. ${ }^{11)}$ In vitro cultured human OSCC cell lines were analyzed for expression of MUC1 using the anti-MUC1 $\mathrm{mAb}$ C595 that recognizes a tumor-associated core protein epitope similar to an anti-MUC1 mAb DF3. Flow cytometry demonstrated expression of MUC1 on OSCC cell lines (Fig. 1). The HSC4 cells expressed a high level of MUC1 on their surfaces. The cell surface MUC1 expression value of the SAS cell line was lower than that of HSC4. The MFI for both HSC3 and HO-1-u-1 cells was less than 10.

Effect of Anti-MUC1 mAb on the Complement Activation and Complement-Mediated Lysis in Vitro Many mAbs may activate the complement system. In this study, OSCC cell lines were treated with C595 along with human serum in order to evaluate the complement activation on these cell surfaces (Fig. 2A). In case of the HSC4 MUC1 


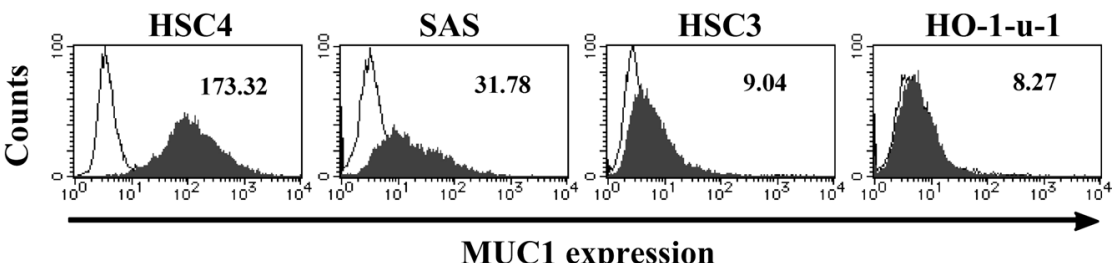

Fig. 1. Analysis of MUC1 Expression on Human OSCC Cell Lines

Relative levels of MUC1 expression determined by flow cytometry with anti-MUC1 C595 mAb. Representative analysis is shown.

(A)
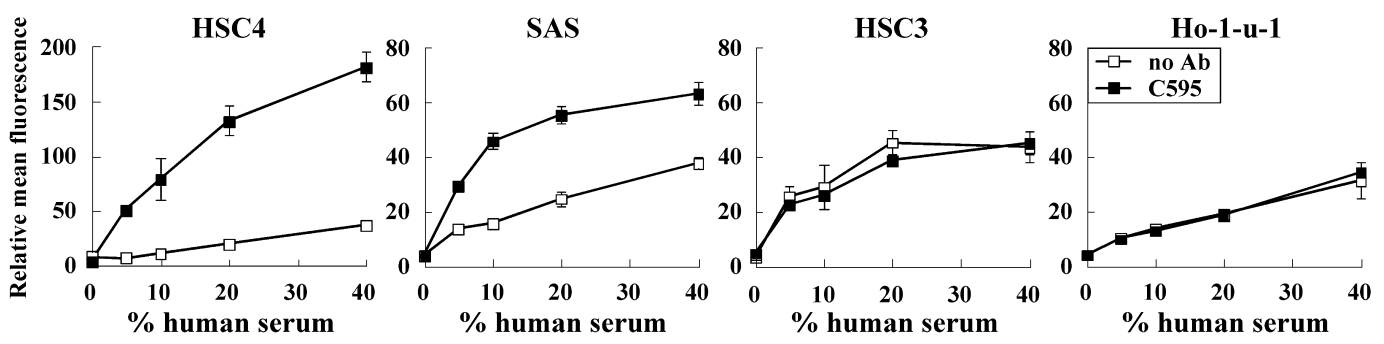

(i)
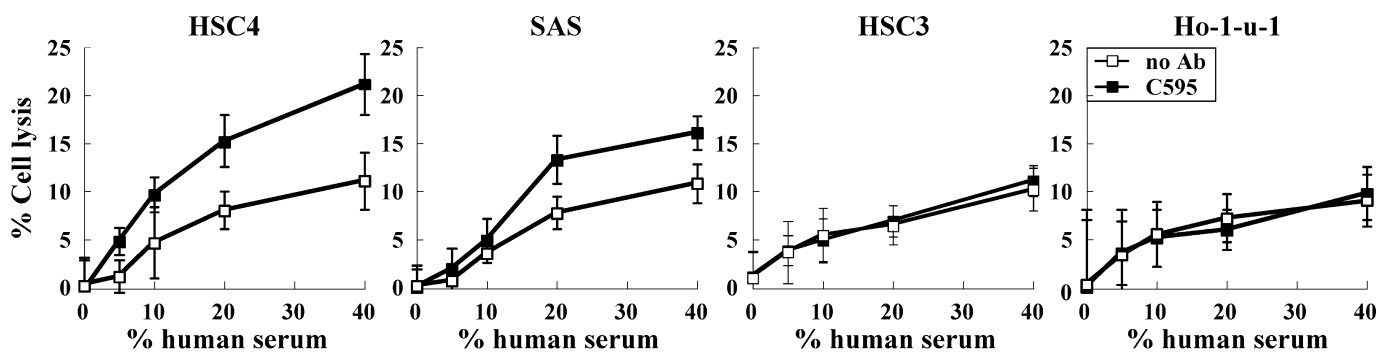

(C)

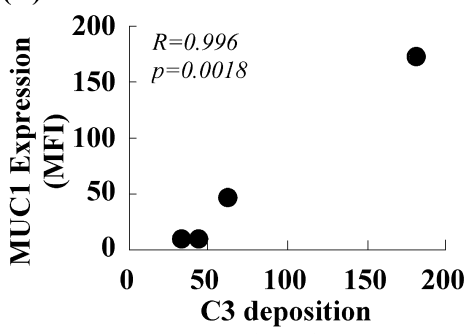

(MFI)
(D)

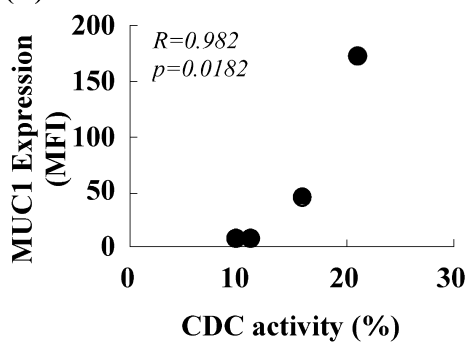

Fig. 2. Effect of Anti-MUC1 Antibody C595 (with Complement Activation) on OSCC

(A) Deposition of human C3 on human OSCC cells. In series (A), cultured OSCC cell lines were preincubated with $100 \mu \mathrm{g} / \mathrm{ml}$ of the anti-MUC1 mAb C595 before incubation in indicated concentration of normal human serum (NHS) as a complement source. Heat inactivated (HI) normal human serum was used as control. C3 deposition was measured by flow cytometry. Data shown is representative of at least 3 trials. (B) Complement-mediated lysis of OSCC cells. OSCC cells were sensitized to complement by preincubation in the indicated concentrations of anti-MUC1 $\mathrm{mAb}$ C595. Sensitized cells were washed out, exposed to normal human serum with the indicated concentration $\left(37{ }^{\circ} \mathrm{C} / 2 \mathrm{~h}\right)$, and cell lysis determined. Figure shows representative data from three separate experiments. (C) Correlation between the expression of MUC1 and the C3 deposition level in the OSCC cancer cell lines. (D) Correlation between the expression of MUC1 and the CDC activity in the OSCC cancer cell lines. The values of MUC1 expression, C3 deposition, and CDC activity were shown for a concentration of $100 \mu \mathrm{g} / \mathrm{ml}$.

high-expression cell line, the administration of C595 with human serum had a highly significant increase of the complement activation compared to no sensitized antibody control. SAS cells also deposited complement in the presence of C595 and NHS. As anticipated, treatment with C595 did not significantly alter the level of $\mathrm{C} 3$ deposition on HSC3 and HO-1-u-1 cells, which had the lowest MUC1 expression. The MFIs at $40 \%$ NHS concentration treated with $100 \mu \mathrm{g} / \mathrm{ml}$ of C595 mAb on HSC4 and SAS cells were 181.23 and 62.82, respectively. Furthermore, C595 activated complement deposition on HSC4 and SAS cells in a human serum and dosedependent manner (Fig. 2A).
We next examined the effect of anti-MUC1 mAb C595 on complement-dependent cell lysis of OSCC cells in vitro (Fig. 2B). Increasing concentrations of human serum led to increased cytotoxicity after $2 \mathrm{~h}$ incubation even without antibody treatment. However, the cell death percentages of OSCC cell lines without C595 were less than 10\%. On HSC4 cells, C595 led to cell killing of approximately $20 \%$ in the presence of NHS, and it was about twice as high when compared to no C595 treatment. C-mediated killing of SAS cells by $\mathrm{C} 595$ was approximately $15 \%$, and lower than that of HSC4 cells. In contrast, HSC 3 and HO-1-u-1 cells were no different than without C595 mAb. EDTA-treated serum and 
heat-inactivated human serum did not lead to killing of these OSCC cell lines in the presence of C595 (data not shown), supporting the hypothesis that the cell killing was mediated via complement activation. We also investigated the correlation between MUC1 expression level and anti-MUC1 mAbmediated complement activation on OSCC cells (Fig. 2C), and CDC activity (Fig. 2D). There were good correlations between MUC1 expression level and C595-mediated C activation $(r=0.996, p=0.0018)$, and between MUC1 expression level and C595-mediated CDC activity $(r=0.982$, $p=0.0182$ ), indicating that the anti-MUC1 $\mathrm{mAb}$ effect to OSCC cells was dependent on MUC1 expression in OSCC cells.

ADCC Activities of C595 Anti-MUC1 mAb in OSCC Cell Lines In order to study whether anti-MUC1 $\mathrm{mAb}$ C595 can induce antibody-dependent cellular cytotoxicity (ADCC) to OSCC cells, we next analyzed C595-mediated ADCC using OSCC cell lines and human peripheral blood mononuclear cells (PBMCs) from normal donors as effector cells (Fig. 3A). In the high MUC1 expression HSC4 cells, $\%$ ADCC activity was approximately $30 \%$ with an effectorto-target ratio of $100: 1$ and $20 \%$ with $\mathrm{E} / \mathrm{T}$ ratio at $25: 1$, and they were significantly different in the absence of C595 $(p<0.0005)$. Similar results were obtained with SAS cells. The percent cell killing by C595-mediated ADCC of SAS cells was approximately $22 \%$ with an effector-to-target ratio of $100: 1$ and it was also significantly different than in the absence of C595 $(p=0.0125)$. In contrast, the MUC1-negative OSCC cell line HO-1-u-1 cells show no inducement of ADCC by $\mathrm{C} 595$. We next examined the relationship between MUC1 expression level and anti-MUC1 mAb-mediated ADCC activity by the comparison of each OSCC cell line (Fig. 3B). The ADCC activity mediated by C595 in HSC4 cells was significantly different than SAS cells $(p=0.0053)$, and the activity in SAS cells was also significantly different than in HO- $1-\mathrm{u}-1$ cells $(p=0.0028)$. In contrast, ADCC activities in HO- $1-\mathrm{u}-1$ cells were $<10 \%$ under all tested conditions and were not different without C595 treatment. MUC1 expression was detected in HSC4 and SAS cells, but not in HO-1-u-1 cells (Fig. 1). Furthermore, ADCC activity mediated by $\mathrm{C} 595$ was highly correlated with the binding values of MUC1 to cells expressing MUC1 in the statistical analysis with the Pearson $\mathrm{R}$ test $(r=0.964, p=0.0005$, data not shown).

Direct Growth Inhibitory Effect of C595 Anti-MUC1 mAb on OSCC Cell Lines Anti-MUC1 mAb C595 showed no growth inhibitory effect in any of the OSCC cell lines examined, regardless of MUC1 expression levels. Even the highest concentration of C595 $(100 \mu \mathrm{g} / \mathrm{ml})$ did not inhibit growth in any of the OSCC cell lines (Fig. 4).

Expression of Complement Regulatory Proteins on OSCC Cell Lines It is well documented that the expression of complement regulatory proteins on tumor cells confers protection from antibody-dependent complement-mediated lysis. In fact, complement-mediated lysis of OSCC cells by $\mathrm{C} 595$ was less than $22 \%$. Although enough levels of antiMUC1 $\mathrm{mAb}$ are deposited on OSCC cells, the increase in the complement activating signals may be insufficient to overcome the effects of complement regulatory proteins. We therefore investigated expression of complement regulatory proteins on OSCC cell lines. All OSCC cell lines express
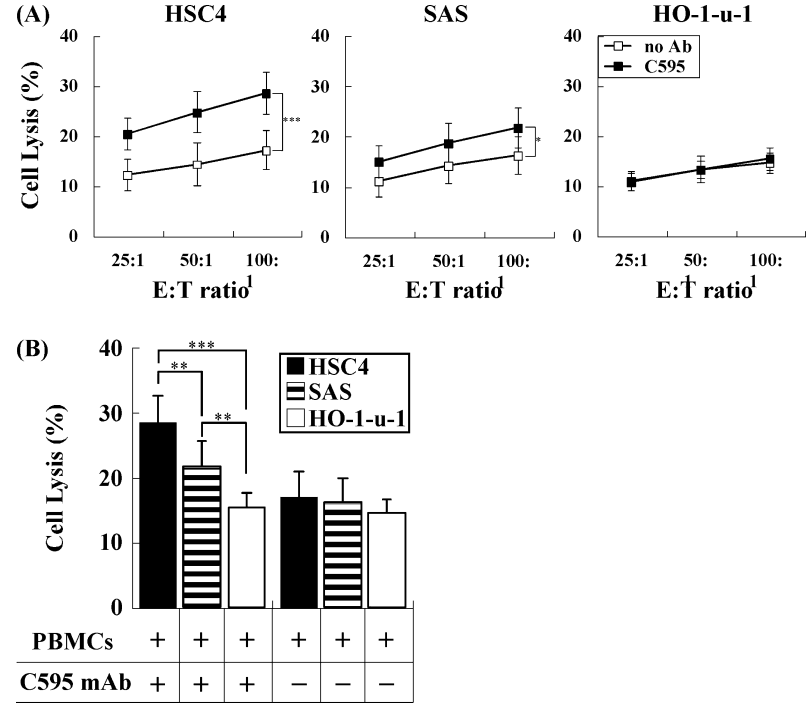

Fig. 3. (A) Antibody-Dependent Cellular Cytotoxicity (ADCC) Activity by the Anti-MUC1 mAb C595 in the OSCC Cell Lines

C595-mediated ADCC activity in OSCC cell lines was determined using the CytoTox 96 Non-Radioactive Cytotoxicity Assay. Mean \pm S.D., $n=8$.

(B) Comparison of C595-Mediated ADCC with Each of the OSCC Cell Lines

ADCC was performed using $100 \mu \mathrm{g} / \mathrm{ml}$ of $\mathrm{C} 595$ and an effector-to-target ratio at $100: 1$

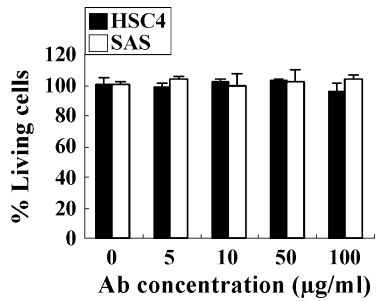

Fig. 4. The Effect of Anti-MUC1 Antibody C595 on Cell Proliferation

Cell proliferation was assessed by MTS assay. Shown is mean \pm S.D. $n=8$ for all experiments.
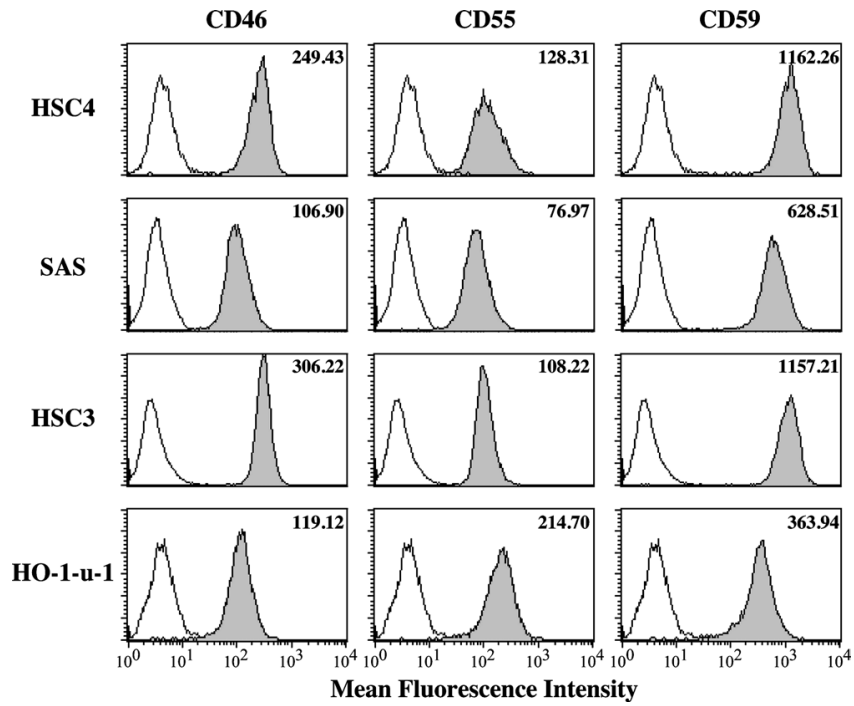

Fig. 5. The Expression of Complement Inhibitory Proteins on Human OSCC Cell Lines

The expression of complement inhibitory proteins examined by flow cytometry using appropriate antibodies. Staining with a control mouse IgG1 antibody gave a mean relative fluorescence of less than 10. Data shown is representative of 2 separate experiments. 
all three membrane-bound complement inhibitors, CD46, CD55, and CD59 (Fig. 5). These data raise the possibility that overexpression of complement regulatory proteins on OSCC cells protects from complement lysis by C595.

\section{DISCUSSION}

Antibody therapies are a major approach in the treatment of various cancer types. In our study, we focused on the antitumor activity of an anti-MUC1 mAb C595 to oral squamous cell carcinomas. Initially, the anti-MUC1 mAb C595 was tested for the ability to bind to three OSCC cell lines. C595 bound to HSC4 cells strongly and to SAS cells moderately. Thus, two-thirds of the OSCC cell lines described in this study expressed MUC1. In OSCC patients, distinct membrane MUC1 mucin staining patterns were identified in $59.7 \% .{ }^{11)}$ Furthermore, the malignant transformation of oral epithelium, tumor invasion, and tumor metastasis were associated with higher MUC1 mucin expression. ${ }^{11)}$ Therefore, our data support the idea that MUC1 is a useful target for immunotherapy of OSCC.

MUC1 is a high-molecular-weight transmembrane glycoprotein, and is highly overexpressed and loses its polarized distribution on the cell surface. ${ }^{14)} \mathrm{MUC1}$ is also underglycosylated, resulting in the exposure of immunodominant peptide sequences that are normally concealed. MUC1 is thus an important marker of malignancy and is a target for several immunotherapies currently under investigation. ${ }^{8)}$ The level of MUC1 expression has been shown to correlate with the degree of breast tumor differentiation, estrogen receptor status, and clinical outcome of breast cancers, ${ }^{9)}$ and both progression and metastases of epithelial ovarian cancers. ${ }^{10)}$ Furthermore, Verhoeyen et al. established a humanized, anti-MUC1 mAb (huHMFG-1). ${ }^{15)}$ HuHMFG-1 induced strong ADCC to breast cancer cells and is currently being used in clinical studies on breast carcinoma. ${ }^{16,17)}$ Herein, we investigated the effect of antibody immunotherapy of anti-MUC1 mAb to oral squamous cell carcinomas, and demonstrated that antiMUC1 mAb C595 was able to kill OSCC cells mediated by $\mathrm{CDC}$ and $\mathrm{ADCC}$, and these effects were strongly correlated with MUC1 expression.

Our data indicated that complement-mediated killing of HSC 4 cells by anti-MUC1 mAb was less than $22 \%$ although ADCC activity was approximately $30 \%$, suggesting that OSCC cells express membrane complement inhibitors. Ravindranath et al. recently reported that membrane complement inhibitors, CD46, CD55 and CD59 were highly expressed in human head and neck cancer cells at T1/ T2/N0/M0 stages. ${ }^{18)}$ They also demonstrated that the relative densities of cell-surface expression of membrane complement inhibitors on OSCCs are as follows: CD59>CD55> CD46 is a pattern found in both breast and pancreatic carcinomas, whereas the profile of membrane complement inhibitors in melanoma $(\mathrm{CD} 59>\mathrm{CD} 55<\mathrm{CD} 46)$ and colon cancer $(\mathrm{CD} 46>\mathrm{CD} 55>\mathrm{CD} 59)$ were different. ${ }^{19)}$ Our data also indicated the similar results (Fig. 5), and therefore OSCC cells could escape from antibody-mediated complement lysis by high expression of membrane complement inhibitors.

Various other approaches to overcome the resistance of tumor cells to antibodies and complement have been investigated in vitro, and the subject is well-reviewed. ${ }^{12,20)}$ Two major approaches to enhance anti-tumor, complement effector mechanisms that have been investigated therapeutically in animal models of cancer are the use of bispecific antibodies and the use of $\beta$-glucan, a bioresponse modifier. Soluble $\beta$ glucan has been used as an adjuvant for mAb therapy, and it induces complement-dependent cellular cytotoxicity by enabling iC $3 \mathrm{~b}$ deposited on tumor cells to activate complement receptor 3 (CR3) on immune effector cells. ${ }^{21,22)}$ Several studies involving different models of cancer have shown that $\beta$ glucan can promote and enhance $\mathrm{mAb}$ therapy, including studies in which $\beta$-glucan was used in combination with antiGD2 and anti-MUC1 mAb therapy. ${ }^{21,22)}$ In the other approach, a bispecific antibody directed against a tumor-associated antigen and Crry, a membrane complement inhibitory protein, significantly inhibited the outgrowth of lung tumors in a rat model of metastatic colorectal cancer. ${ }^{23)}$ As another approach, dexamethasone has been shown to upregulate MUC1 expression on certain types of cancer cell lines in vitro, suggesting that this gluocorticoid may enhance MUC1based immunotherapies. ${ }^{24)}$ Recently, CR2-Fc was reported as a novel fusion protein for supporting antibody immunotherapy. CR2-Fc is able to both enhance C3 deposition with increased complement-mediated lysis and ADCC. ${ }^{25)}$ The strategy may be additive to all $\mathrm{mAb}$ effector mechanisms, including those that do not have ADCC and complement cytotoxicity as a recognized component of their primary mechanism of action. ${ }^{25}$ Combination of these tools with anti-MUC1 $\mathrm{mAb}$ therapy may overcome OSCC and contribute to the development of strong effect and low side-effect therapy for OSCC.

In summary, there is increasing interest in the use of unconjugated mAbs to treat cancer; several have been approved for therapy and several more are under investigation in the laboratory and in the clinic. Their mechanisms of action appear to be variable, but evidence from animal models and from clinical studies indicate that ADCC and complementdependent cytotoxicity play an important role in the efficacy of many mAbs. In this study, we demonstrate the potential of an immunotherapeutic strategy against OSCC with antiMUC1 mAb. The approach may be clinically viable to the management of OSCC in the future.

Acknowledgments We thank Mr. Bryan J. Mathis for the English editing of this manuscript. This work was supported by Grants-in-Aid from the Ministry of Education, Culture, Sports, Science and Technology of Japan.

\section{REFERENCES}

1) Jemal A., Siegel R., Ward E., Murray T., Xu J., Thun M. J., CA. Cancer J. Clin., 57, 43-66 (2007).

2) Shintani S., Mihara M., Li C., Nakahara Y., Hino S., Nakashiro K., Hamakawa H., Cancer Sci., 94, 894-900 (2003).

3) Sasabe E., Zhou X., Li D., Oku N., Yamamoto T., Osaki T., Int. J. Cancer, 120, 268-277 (2007).

4) Shen J., Huang C., Jiang L., Gao F., Wang Z., Zhang Y., Bai J., Zhou H., Chen Q., Biochem. Pharmacol., 73, 1901-1909 (2007).

5) Thomas G. R., Chen Z., Enamorado I., Bancroft C., Van Waes C., Int J. Cancer, 86, 368-374 (2000).

6) Suwa T., Saio M., Umemura N., Yamashita T., Toida M., Shibata T., Takami T., Oncol. Rep., 15, 757-763 (2006).

7) Reichert T. E., Scheuer C., Day R., Wagner W., Whiteside T. L., Cancer, 91, 2136-2147 (2001). 
8) Denda-Nagai K., Irimura T., Glycoconj. J., 17, 649-658 (2000).

9) Hayes D. F., Mesa-Tejada R., Papsidero L. D., Croghan G. A., Korzun A. H., Norton L., Wood W., Strauchen J. A., Grimes M., Weiss R. B., J. Clin. Oncol., 9, 1113-1123 (1991).

10) Wang L., Ma J., Liu F., Yu Q., Chu G., Perkins A. C., Li Y., Gynecol. Oncol., 105, 695-702 (2007).

11) Nitta T., Sugihara K., Tsuyama S., Murata F., Cancer, 88, 245-254 (2000).

12) Gelderman K. A., Tomlinson S., Ross G. D., Gorter A., Trends Immunol., 25, 158-164 (2004).

13) Yu J., Abagyan R., Dong S., Gilbert A., Nussenzweig V., Tomlinson S., J. Exp. Med., 185, 745-753 (1997).

14) Ho S. B., Niehans G. A., Lyftogt C., Yan P. S., Cherwitz D. L., Gum E. T., Dahiya R., Kim Y. S., Cancer Res., 53, 641-651 (1993).

15) Verhoeyen M. E., Saunders J. A., Price M. R., Marugg J. D., Briggs S., Broderick E. L., Eida S. J., Mooren A. T., Badley R. A., Immunology, 78, 364-370 (1993).

16) Snijdewint F. G., von Mensdorff-Pouilly S., Karuntu-Wanamarta A. H., Verstraeten A. A., Livingston P. O., Hilgers J., Kenemans P., Int. J.
Cancer, 93, 97-106 (2001).

17) Moreno M., Bontkes H. J., Scheper R. J., Kenemans P., Verheijen R. H., von Mensdorff-Pouilly S., Cancer Lett., 257, 47-55 (2007).

18) Ravindranath N. M., Shuler C., J. Oral Pathol. Med., 35, 560-567 (2006).

19) Ravindranath N. M., Shuler C., Oral Surg. Oral Med. Oral Pathol. Oral Radiol. Endod., 103, 231-239 (2007).

20) Macor P., Tedesco F., Immunol. Lett., 111, 6-13 (2007).

21) Hong F., Yan J., Baran J. T., Allendorf D. J., Hansen R. D., Ostroff G. R., Xing P. X., Cheung N. K., Ross G. D., J. Immunol., 173, 797-806 (2004).

22) Cheung N. K., Modak S., Clin. Cancer Res., 8, 1217-1223 (2002).

23) Gelderman K. A., Kuppen P. J., Okada N., Fleuren G. J., Gorter A., Cancer Res., 64, 4366-4372 (2004).

24) Imai M., Hwang H. Y., Norris J. S., Tomlinson S., Immunology, 111, $291-297$ (2004).

25) Imai M., Ohta R., Varela J. C., Song H., Tomlinson S., Cancer Res., 67, 9535-9541 (2007) 